MEMBRANE DYNAMICS

\title{
ER marks the spot
}

The endoplasmic reticulum (ER) and mitochondria are known to be intimate, showing stable contacts that can affect lipid synthesis and calcium signalling between the two organelles. The Voeltz laboratory, in collaboration with the Nunnari laboratory, has followed the relative dynamics of these organelles more closely and found that ER contact with mitochondria may actively trigger mitochondrial constriction and subsequent division.

The authors first used electron microscopy and tomography to get a high-resolution and three-dimensional view of how the ER and mitochondria interact in the budding yeast Saccharomyces cerevisiae. This revealed that ER tubules not only make contact with mitochondria but can also wrap around them, sometimes entirely, and this correlated with sites of mitochondrial constriction.

The groups next turned to live cells to follow the dynamics of ER-mitochondria interplay. In both yeast and mammalian cells, similar interfaces between the two organelles were observed and, again, these coincided with sites of constriction and subsequent division. Interestingly, the shape and architecture of the ER at contact sites seemed to be very important, as division occurred predominantly at positions where ER tubules wrapped around the mitochondrial membrane.

Next, they asked how these sites of contact relate to factors that are known to regulate mitochondrial division. Mitochondrial structure is maintained by balancing division and fusion events. Dynamin-related protein 1 (DRP1; known as Dnm1 in yeast) is a key regulator of mitochondrial fission and is conserved from yeast to mammals. Through live imaging of three fluorescent markers for the ER, the mitochondria and DRP1 (or Dnm1), they saw that the sites of ER-mitochondrial contact showed significant colocalization with DRP1 (or Dnm1) punctae in both yeast and mammalian cells, and many of these also associated with sites of mitochondrial constriction. Thus, they conclude that ER contact with mitochondria has an instructive role in mitochondrial division. Furthermore, constrictions formed at ER contact sites in the absence of DRP1 and even mitochondrial fission factor (MFF), which regulates DRP1 recruitment, suggesting that the ER may function to help constrict the mitochondria before these division factors are recruited. This also raises the interesting possibility that ER-mediated constriction of mitochondria may provide the initial reduction in membrane diameter that then allows DRP1 helices to form and promote fission.

Thus, the ER can determine the shape of a neighbouring organelle. By marking the point at which mitochondrial fission will take place, the ER has a key role in regulating the balance of mitochondrial dynamics. This may have important implications for diseases in which mitochondrial structure is perturbed and for normal cellular functions, such as cell death.

Alison Schuldt

ORIGINAL RESEARCH PAPER Friedman, J. R. et al. ER tubules mark sites of mitochondrial division. Science 1 Sep 2011 (doi:10.1126/science.1207385)
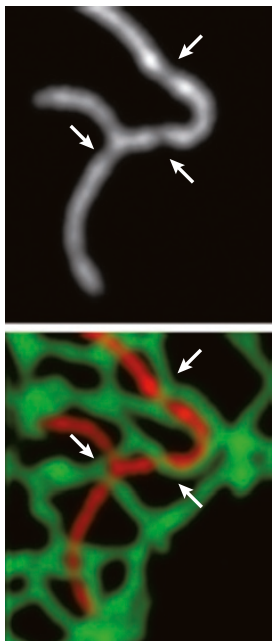

Image of a mitochondrion alone (top) and merged with an image of the endoplasmic reticulum (ER; bottom) in COS7 cell depleted of mitochondrial fission factor by RNA interference. Arrows mark sites of mitochondrial constriction at crossing ER tubules. Images courtesy of J. Friedman, University of Colorado at Boulder, USA 